\section{Das pathogene immunologische Gedächtnis in der chronischen Entzündung}

\author{
A. Radbruch ${ }^{1,2}$ \\ 1 Deutsches RheumaForschungsZentrum Berlin, \\ ein Leibniz Institut \\ ${ }^{2}$ Charité Universitätsmedizin, Humboldt Universität Berlin
}

Was treibt eine chronische rheumatische Entzündung an? Ist es eine chronische Immunreaktion gegen Autoantigene oder persistierende Infektionserreger? Oder entwickelt das Immunsystem ein Gedächtnis, das die Entzündung unabhängig von Antigenen unterhält, und auch nach therapeutischer Intervention wieder aufflammen lassen kann? Wenn es ein solches Gedächtnis gibt, brauchen wir dann nicht ganz neuartige Therapien, um dieses pathogene Gedächtnis zu löschen und die chronische Entzündung dauerhaft zu beenden? Wir müssten die pathogenen Zellen des immunologischen Gedächtnisses ausschalten.

Was spricht dafür, dass es ein pathogenes immunologisches Gedächtnis gibt, das eine chronische Entzündung antreibt? Da gibt es zunächst einmal die Beobachtung, dass rund 70\% der Patienten mit chronisch-entzündlichen Erkrankungen, die nicht auf herkömmliche Therapien ansprechen, in eine langfristige Therapie-freie Remission gehen, wenn ihr Immunsystem zerstört und aus körpereigenen Stammzellen wieder aufgebaut wird. Bei dieser Therapie der „Immunablation mit autologer Stammzelltransplantation" (ASCT) wird im Wesentlichen das immunologische Gedächtnis gelöscht. In den Patienten entwickelt sich ein neues, naives Immunsystem, das dem von Kindern gleicht [1]. Es ist tolerant und greift den eigenen Körper nicht mehr an, obwohl die von der chronischen Entzündung betroffenen Gewebe noch nicht regeneriert und durch eine Barriere vor dem Immunsystem geschützt sind. Diese Beobachtung spricht also dagegen, dass die chronische Entzündung durch eine chronische Immunreaktion angetrieben wurde, und dafür, dass es ein pathogenes Gedächtnis war, das sie angetrieben hat.

Wenn pathogene Gedächtniszellen eine chronische Entzündung treiben, kann man dann mit Gedächtniszellen eine chronische Entzündung auslösen? Gezeigt wurde das für Gedächtnis-Plasmazellen in einem experimentiellen Mausmodell. Pathogene Gedächtnis-Plasmazellen sezernieren Autoantikörper, die körpereigenes Gewebe für die Effektorzellen der Entzündung markieren, also für Granulozyten und Makrophagen. In NZB/W Mäusen, einem Modell für Systemischen Lupus Erythematosus (SLE), sezernieren die pathogenen Gedächtnis-Plasmazellen Autoantikörper, die zu einer Entzündung der Niere führen. Durch eine Übertragung von Gedächtnis-Plasmazellen aus NZB/W Mäusen in gesunde Mäuse konnte die Arbeitsgruppe von Falk Hiepe in den gesunden Mäusen eine chronische Nierenentzündung auslösen, die der in NZB/W Mäusen glich [4]. Gedächtnis-Plasmazellen, die pathogene Autoantikörper sezernieren, können also eine chronische Entzündung auslösen und unterhalten.
Dies Ergebnis ist von besonderer therapeutischer Relevanz, weil Gedächtnis-Plasmazellen in besonderen Nischen des Knochenmarks überleben. Sie sprechen nicht auf konventionelle Therapien an, sezernieren weiter ihre Autoantikörper. Neue therapeutische Strategien zielen auf Gedächtnis-Plasmazellen [8]. Gerade wurde eine erste Studie mit dem Proteasomenblocker Bortezomib abgeschlossen, in der bei Patienten mit SLE eindrucksvolle Erfolge erzielt wurden [2]. Idealerweise würde man selektiv die Plasmazellen eliminieren, die Autoantikörper sezernieren, und nicht alle Plasmazellen, damit auch die, die schützende Antikörper sezernieren. Ein möglicher Ansatz wurde gerade von der Arbeitsgruppe von Falk Hiepe berichtet [14].

Die Rolle von Gedächtnis-B- und -T-Lymphozyten als Treiber chronischer Entzündungen ist weit weniger klar als die Rolle der Gedächtnis-Plasmazellen. Der therapeutische Erfolg von anti-CD20 (Rituximab), das selektiv zirkulierende B-Lymphozyten eliminiert, bei Patienten mit chronischen Entzündungen deutet auf eine Rolle der B-Lymphozyten als wichtige Antigen-präsentierende und immunregulierende Zellen hin [12]. Allerdings führt Rituximab bei den meisten Patienten nicht in die Therapie-freie Remission. Es reicht also offenbar nicht, die zirkulierenden Gedächtnis-B-Lymphozyten zu eliminieren. Noch unklarer ist die Lage bezüglich der Gedächtnis-T-Lymphozyten. Obwohl es eine Reihe experimenteller Tiermodelle gibt, bei denen eine chronische Entzündung nur von T-Lymphozyten eingeleitet und getrieben wird, wird die Rolle pathogener Gedächtnis-T-Lymphozyten in menschlichen Krankheiten kontrovers diskutiert [3]. Interessanterweise passen sich aktivierte T-Lymphozyten an eine wiederholte Aktivierung an. In einer chronischen Entzündung exprimieren sie neue Gene, wie den Transkriptionsfaktor Twist1. Twist1 unterdrückt die Expression von entzündungsfördernden Genen, ein endogener Dämpfer der Entzündung [11]. Twist1 sorgt aber auch dafür, dass die pathogenen Gedächtnis-T-Zellen am Leben bleiben, indem es die Expression der MikroRNA-148a induziert, die ihrerseits das proapoptotische Protein Bim unterdrückt [6]. Twist1 sorgt also gleichsam für „Chronizität“. Die meisten pathogenen Gedächtnis-T-Lymphozyten exprimieren auch den Transkriptionsfaktor T-bet, der seinerseits die Expression proinflammatorischer Zytokine induziert, z.B. die Expression von Interferon-gamma. T-bet sorgt auch dafür, dass die Gene für die pro-inflammatorischen Zytokine epigenetisch geprägt werden, sodass sie bei späteren Aktivierungen automatisch exprimiert werden [5,7, 10]. Dieses „Zytokingedächtnis“ ist ein wesentliches Merkmal der proinflammatorischen Gedächtnis-T-Lymphozyten. Allerdings unterdrückt T-bet auch die Expression des Zytokins Interleukin 2 (IL-2) [13]. Das hat Konsequenzen für die Regulierung von Immunantworten, denn regulatorische T-Lymphozyten, die Immunantworten abschalten können, benötigen IL-2 von aktivierten T-Lymphozyten, um selber aktiv zu werden. Pathogene Gedächtnis-T-Lymphozyten entziehen sich so der physiologischen Immunregulation. Kann man also durch therapeutisches IL-2 die physiologische Immunregulation aktivieren und pathogene Gedächtnis-T-Lymphozyten unterdrücken? Erste Ergebnisse einer experimentellen Therapie eines Patienten mit SLE deuten darauf hin, dass das möglicherweise funktioniert [9]. Es bleibt festzuhalten, dass 
bisher nur die ASCT-Therapie, bei der das gesamte immunologische Gedächtnis gelöscht wird, zur Induktion langfristiger Therapie-freier Remission geführt hat. Die selektive Eliminierung von Gedächtnis-B-Lymphozyten (Rituximab), Gedächtnis-Plasmazellen (Bortezomib) oder die Aktivierung der Inhibition von Gedächtnis-T-Lymphozyten (IL-2) kann das offenbar nicht, trotz aller klinischen Wirksamkeit. Es scheint, dass das immunologische Gedächtnis so redundant organisiert ist, dass man verschiedene Typen von Gedächtniszellen gleichzeitig eliminieren muss, um es zu löschen. Welche Kombinationen man braucht und wie man dabei das schützende immunologische Gedächtnis erhalten kann, das sind die gegenwärtigen Herausforderungen an die Forschung. Interessenkonflikt: Es bestehen keine Interessenkonflikte.

\section{Literatur}

1 Alexander T, Thiel A, Rosen $O$ et al. Depletion of autoreactive immunologic memory followed by autologous hematopoietic stem cell transplantation in patients with refractory SLE induces long-term remission through de novo generation of a juvenile and tolerant immune system. Blood 2009; 113: 214-223

2 Alexander T, Sarfert R, Klotsche J et al. The proteasome inhibitior bortezomib depletes plasma cells and ameliorates clinical manifestations of refractory systemic lupus erythematosus. Ann Rheum Dis 2015; 74: $1474-1478$

3 Chang HD, Radbruch A. Targeting pathogenic T helper cell memory. Ann Rheum Dis 2011; 70 (Suppl. 1): i85-i87

4 Cheng $Q$ Mumtaz IM, Khodadadi L et al. Autoantibodies from long-lived 'memory' plasma cells of NZB/W mice drive immune complex nephritis. Ann Rheum Dis 2013; 72: 2011 - 2017

5 Dong J, Chang HD, Ivascu C et al. Loss of methylation at the IFNG promoter and CNS- 1 is associated with the development of functional IFN-E $\geq$ memory in human CD4(+) T lymphocytes. Eur J Immunol 2013; 43: 793-804

6 Haftmann C, Stittrich AB, Zimmermann J et al. miR-148a is upregulated by Twist 1 and T-bet and promotes Th1-cell survival by regulating the proapoptotic gene Bim. Eur J Immunol 2015; 45: 1192 - 1205
7 Helmstetter C, Flossdorf M, Peine $M$ et al. Individual T helper cells have a quantitative cytokine memory. Immunity 2015; 42: 108-122

8 Hiepe F, Dörner T, Hauser AE et al. Long-lived autoreactive plasma cells drive persistent autoimmune inflammation. Nat Rev Rheumatol 2011; 7: $170-178$.

9 Humrich JY, von Spee-Mayer C, Siegert E et al. Rapid induction of clinical remission by low-dose interleukin-2 in a patient with refractory SLE. Ann Rheum Dis 2015; 74: 791 - 792

10 Mazzoni A, Santarlasci V, Maggi L et al. Demethylation of the RORC2 and IL17A in human CD4+ T lymphocytes defines Th17 origin of nonclassic Th1 cells. J Immunol 2015; 194: 3116-3126

11 Niesner U, Albrecht I, Janke M et al. Autoregulation of Th1-mediated inflammation by twist1. J Exp Med 2008; 205: 1889-1901

12 Shen P, Fillatreau S. Antibody-independent functions of B cells: a focus on cytokines. Nat Rev Immunol 2015; 15: 441 - 451

13 Szabo SJ, Kim ST, Costa GL et al. A novel transcription factor, T-bet, directs Th1 lineage commitment. Cell 2000; 100: 655-669

14 Taddeo A, Gerl V, Hoyer BF et al. Selection and depletion of plasma cells based on the specificity of the secreted antibody. Eur J Immunol 2015; 45: $317-319$

Bibliografie

DOI http://dx.doi.org/10.1055/s-0035-1558065

Drug Res 2015; 65, Suppl. 1: S16-S17

(c) Georg Thieme Verlag KG Stuttgart · New York .

ISSN 2194-9379

Korrespondenzadresse

Prof. Dr. Andreas Radbruch

Deutsches Rheuma-Forschungszentrum (DRFZ)

Charitéplatz 1

10117 Berlin

radbruch@drfz.de

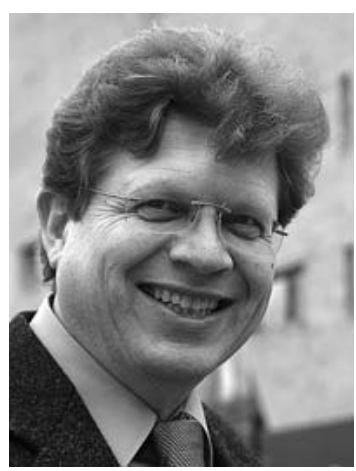

